\title{
Los peajes urbanos como factor determinante de sostenibilidad y competitividad en el transporte urbano: un estudio aplicado a Madrid
}

Juan Muñoz. Universidad Rey Juan Carlos, Madrid, España.

Francisca Anguita. Universidad Rey Juan Carlos, Madrid, España.

RESUMEN | Los sistemas de peaje urbano constituyen una medida altamente contributiva a la mejora de la sostenibilidad en el transporte en las grandes ciudades. El presente artículo ofrece una valoración y análisis de los cambios en el comportamiento de movilidad de los ciudadanos de Madrid como consecuencia de la implantación de un hipotético peaje en el centro urbano. Las conclusiones alcanzadas, de acuerdo con los resultados obtenidos a través de una encuesta poblacional aplicada a 1.298 ciudadanos, nos ha permitido determinar que la aplicación de esta medida proporcionaría una importante reducción del uso del automóvil, un aumento considerable del uso del transporte público y otros modos más sostenibles (caminar o bicicleta), y una significativa disminución de la congestión urbana en el centro de la ciudad y su entorno, lo que contribuiría a alcanzar un mayor grado de sostenibilidad en el transporte para la región en su conjunto.

PALABRAS ClAVE | movilidad, sustentabilidad urbana, transporte urbano.

ABSTRACT | Urban road pricing is a highly contributory measure to improve sustainability in transport in the area of large cities. This article provides an assessment and analysis of changes in the mobility behavior of the citizens of Madrid as a result of the implementation of a hypothetical toll in the city center. According to the results obtained through a population survey of 1,298 citizens, the study concludes that the application of this measure would provide a significant reduction in car use, a considerable increase in the use of public transport and other sustainable modes (walking or cycling) and a significant reduction of urban congestion in the city center and its surroundings, which would help to achieve greater sustainability in transport for the region as a whole.

KEYWORDS | mobility, urban sustainability, urban transportation. 


\section{Introducción}

El incremento progresivo del tráfico motorizado en las grandes ciudades y su entorno, junto con ser causa de numerosos problemas económicos, sociales y medioambientales, da lugar a elevados niveles de congestión, principalmente debido al uso masivo del automóvil privado como modo de transporte habitual en los desplazamientos urbanos. Ante esta situación, determinadas políticas de transporte urbano, como la implantación de zonas de bajas emisiones (Wolff, 2014), la restricción al tráfico en el interior de la ciudad en función del número de placa (Wang, Xu \& Qin, 2014) o la tarificación vial, y más concretamente los peajes urbanos (Anas \& Linsey, 2011; Eliasson, Hultkrantz, Nerhagen \& Rosqvist, 2009; Schade, 2005; Schade \& Schlag, 2003), se presentan como medidas efectivas frente a la congestión. La estipulación de peajes urbanos supone una fórmula para regular o limitar el uso o acceso a un recurso escaso, como es la infraestructura vial urbana existente (Pigou, 1920), mediante un cordón de peaje que regule la congestión circulatoria (Vickrey, 1963; Walters, 1961). En este sentido, el peaje urbano impone a los usuarios de movilidad el pago de una tarifa cuando utilicen su automóvil dentro de un área específica (Jaensirisak, Wardman \& May, 2005).

El establecimiento de una medida como el peaje urbano se justifica por su efectividad; esto es, su alta capacidad para alcanzar objetivos como la reducción de la congestión, la mejora del medioambiente, de la salud, seguridad y habitabilidad urbana, además de permitir una fuente de ingresos adicional para inversiones en transporte sostenible (Curacao, 2009a). Tras su puesta en funcionamiento, los principales peajes urbanos de anticongestión revelaban que los objetivos mencionados pueden ser logrados con éxito. En el caso de Singapur, donde el sistema de peaje opera desde mediados de los setenta, tras su puesta en marcha se redujo la entrada de vehículos en el centro urbano en un $73 \%$ durante el horario de funcionamiento (Holland \& Watson, 1978); el peaje londinense (Santos \& Fraser, 2006; Leape, 2006) contribuyó a una reducción de $18 \%$ en el número total de vehículos entrantes en la zona de peaje en horario tarificado (Transport for London [TfL], 2004); el peaje de Estocolmo, tras su implantación, supuso una reducción de la congestión próxima al 25\% (Eliasson, 2008); por último, está la experiencia más reciente de Milán, donde se observó una reducción de la congestión en torno al 13,2\% en el interior del cordón (Rotaris, Danielis, Marcucci \& Massiani, 2010). En todos los casos, la reducción de tráfico estuvo acompañada de una mejora de los tiempos de desplazamiento y un efectivo aumento del uso del transporte público -la fiabilidad del transporte público en superficie en el caso de Londres mejoró un 40\% (TfL, 2008), además de una mejora de la calidad medioambiental (Banister, 2003)-. No obstante tales beneficios, esta medida encuentra un importante obstáculo en su baja aceptabilidad social, debido a su incidencia sobre la pérdida de equidad en la movilidad para los ciudadanos (Eriksson, Garvill \& Nordlund, 2006) y los efectos regresivos de exclusión para las clases más modestas en sus desplazamientos habituales (Schlag \& Teubel, 1997), al proporcionar beneficios solo a aquellos individuos que tienen una capacidad económica más elevada para soportar el pago de la tarifa de peaje (Giuliano, 1994). 
En este contexto, centrando nuestra atención en los efectos sobre la reducción de la congestión que potencialmente permite esta medida y sin entrar a abordar la problemática de la aceptabilidad y equidad social, el objetivo de nuestra investigación es valorar y analizar de forma cuantitativa y cualitativa los principales impactos que se deducirían de la implantación de un peaje urbano en Madrid. Nuestro análisis ha consistido en un estudio empírico realizado a través de una encuesta poblacional aleatoria para los habitantes que efectúan desplazamientos de carácter habitual en la región de Madrid. El estudio ha sido planteado bajo el supuesto de un hipotético cordón de peaje en torno a la vía de circunvalación M-30, que circunscribe el centro urbano. Un análisis preliminar del contexto actual de movilidad en Madrid en relación con el grado de congestión urbana, su tipología y el grado de accesibilidad del sistema de transporte público y su competitividad como alternativa al automóvil privado, nos ha permitido determinar la justificación que podría aducirse para la aplicación de dicha medida. Las conclusiones de nuestro estudio revelan impactos muy positivos que inciden sobre una mejora considerable de la sostenibilidad de la movilidad en la ciudad, como son una mayor utilización del transporte público y los modos más sostenibles (caminar o ir en bicicleta), una importante reducción en el uso del automóvil en los desplazamientos habituales, una significativa reducción de la congestión en el centro urbano y su entorno, y la no existencia a priori de efectos adversos relevantes como consecuencia de la implantación del sistema.

\section{El contexto de movilidad urbana en Madrid y su entorno}

\section{El grado de congestión urbana y la necesidad de tarificación vial como solución factible}

El crecimiento de la población que afecta de forma generalizada a las grandes ciudades, asociado al fenómeno de la deslocalización tanto residencial como de actividades empresariales hacia coronas metropolitanas cada vez más alejadas del centro urbano, ha intensificado el uso masivo del automóvil, potenciado además por un exceso de infraestructuras viales y una cultura de movilidad basada en la mayor autonomía y libertad ${ }^{1}$ que ofrece el vehículo propio en los desplazamientos. Esta situación, en el caso concreto de Madrid, ha contribuido a consolidar altos niveles de congestión urbana, que podríamos denominar estructural, debido a su carácter progresivo y permanente en el tiempo, a pesar del desarrollo espectacular que ha experimentado el transporte público tanto urbano como interurbano (Muñoz, 2012). Las políticas expansivas basadas en el aumento de infraestructuras viales (carreteras), fundamentalmente vías de carácter orbital en torno a la ciudad de Madrid, ${ }^{2}$ más que solucionar el problema de la congestión lo ha aumentado, como

1 El usuario de automóvil privado tenderá a utilizar este modo aunque le suponga incrementar tiempos de desplazamiento o soportar elevados costes operacionales de su uso en lugar de utilizar el transporte público, fundamentalmente por razones de flexibilidad horaria y mayor privacidad (Thompson, 1997).

2 Madrid, además del primer cinturón de circunvalación denominado M-30, que rodea el centro de la ciudad, dispone de tres cinturones de circunvalación adicionales ( $\mathrm{M}-4 \mathrm{O}, \mathrm{M}-45$ y M-50), que orbitan de forma concéntrica respecto al primer cinturón. 
consecuencia del denominado 'efecto llamada al uso del automóvil', que provoca un incremento significativo del número de viajes en términos de espacio y tiempo, haciendo que el nivel de congestión no solo se mantenga, sino que incluso se intensifique notablemente (Mogridge, 1986; Mogridge \& Holden, 1987). El panorama descrito, de acuerdo con De Grange y Troncoso (2015), justifica la tarificación vial, que ofrece beneficios potenciales en términos de reducción de tráfico, al aplicar una tarifa donde se prevé congestión futura -como es el centro urbano- y, sobre esa base, trasladar el exceso circulatorio a espacios viales que no presentan elevados niveles de tráfico.

Concretamente, las principales causas de congestión en Madrid son el crecimiento poblacional y el incremento del parque automovilístico. En relación con el crecimiento poblacional experimentado en los últimos ańos, cabe destacar que, para el período 2001-2010, la corona metropolitana y el resto de municipios más alejados del centro urbano han crecido en torno a un $29,53 \%$ y un $48,17 \%$, respectivamente, mientras que el centro urbano ha incrementado su población, por término medio, en un 18,66\% (Muńoz, 2012). Esta pérdida de peso poblacional del centro de la ciudad con respecto a la periferia es consecuencia de la dispersión residencial que, unida al elevado poder de atracción que aún conserva la zona urbana central para organizar buena parte del territorio metropolitano, intensifica la movilidad entre la periferia y dicha zona central (Bayona \& Pujadas, 2014). Por su parte, la evolución del parque automovilístico refleja un aumento de un 3,22\% y un 19,45\% para automóviles y camiones, respectivamente, durante el mismo periodo 20012010, de forma que la ocupación vial y la intensidad de circulación en el centro de la ciudad y su entorno más próximo se han visto incrementadas en un 11,54\% y un 36,36\%, respectivamente (Muńoz, 2012). Estas causas y su tendencia creciente en el tiempo, unidas a un factor subjetivo como es la marcada preferencia en el uso del automóvil por razón de estatus o prestigio, hacen que aumente el tráfico circulatorio, fundamentalmente en horas punta (Thomson \& Bull, 2002), y plantean con fuerza la necesidad de medidas efectivas basadas en la tarificación vial.

La Fundación RACC (2009) realizó un estudio para la ciudad de Madrid en el que se obtuvieron importantes resultados en relación con la congestión y su coste. Según este estudio, el tiempo perdido para el conjunto de los usuarios afectados por día tipo se estima en 329.000 horas (81,2 millones de horas/año), correspondiendo dicha pérdida de tiempo, en un 67\%, a desplazamientos que tienen lugar en las carreteras radiales con destino-origen en el centro urbano, donde el 62,9\% de dicho tiempo perdido se produce en horas punta. Respecto a la infraestructura viaria congestionada, el estudio indica que, por término medio, un $84 \%$ de la congestión se concentra en tan solo 3\% de la red viaria por día tipo durante los periodos de hora punta comprendidos entre las 7:00 y 10:00 horas y las 19:00 y 20:00 horas. La distribución del coste total de congestión pone de manifiesto que, del coste total estimado, el $62 \%$ corresponde al transporte privado, frente al $29 \%$ que se atribuye al transporte público. Por último, el $7 \%$ y $2 \%$ del coste total restante correspondería a costes de carburante y emisiones de gases de efecto invernadero, indicadores que se traducen en unos costes de congestión en los accesos a Madrid de 3,4 millones de 
euros al día, equivalentes a 839 millones de euros al año, todo ello sin considerar los costes derivados de la contaminación atmosférica.

\section{El grado de accesibilidad en el sistema de transporte público urbano como garantía de aceptabilidad social de un peaje urbano}

La tarifa de peaje que soporta el usuario del automóvil, además de absorber los costes marginales sociales -externalidades negativas que el usuario genera socialmente cuando utiliza el vehículo y provoca congestión-, contribuye a una reducción del uso del mismo, lo que supone una reducción de los tiempos de desplazamiento del transporte público en superficie (autobuses). Con ello se consigue un traslado de la demanda a este modo de transporte, que incidiría en un aumento de la frecuencia del servicio en la red de autobuses. Bajo este supuesto, se puede afirmar que el peaje urbano aumentaría la rentabilidad y eficiencia del sistema de transporte público en superficie (Thomson, 1982). No obstante, este efecto positivo requiere, como punto de partida, un sistema de transporte público ampliamente desarrollado y accesible. Solo así se puede evitar que surjan problemas de aceptabilidad social ante una medida como el peaje por parte de los distintos grupos de usuarios que, por razones económicas, puedan verse afectados por ella en caso de no existir alternativas competitivas y accesibles de transporte público (Bonsall \& Kelly, 2005). Un claro ejemplo de lo expuesto es la elevada aceptación social mediante referéndum en el caso del peaje de Estocolmo, como consecuencia de la mejora de la fiabilidad y los tiempos de desplazamiento o velocidad comercial del transporte en superficie durante el periodo de prueba previo a su aceptación (Pozueta 2008). Por lo tanto, la aceptación social progresiva de los peajes urbanos se encuentra vinculada a dos aspectos clave: en primer lugar, la existencia de un sistema de transporte público suficientemente desarrollado, accesible y de calidad, como alternativa eficiente ante la obligación de pagar por circular en automóvil; y en segundo lugar, que el usuario de movilidad perciba en todo momento que la inversión de los fondos procedentes del peaje se emplean de forma real y efectiva en el desarrollo y potenciación del transporte público (Albalate \& Bel, 2009), lo que proporciona mayor motivación ciudadana hacia la aceptación del sistema (Ubbels \& Verhoef, 2004).

La premisa básica de un sistema de transporte público competitivo y accesible como alternativa sostenible al uso del automóvil, se configura actualmente como una pregunta de difícil respuesta, ya que supone considerar la valoración subjetiva del usuario que busca maximizar la utilidad en ese modo de transporte para decidir si abandona el automóvil en sus desplazamientos habituales. Al respecto, algunos autores como Hensher y Prioni (2002) indican que el motivo de viaje no influye en el uso del transporte público, mientras que la edad y la renta son dos atributos decisivos, opinión compartida por Bamberg, Rölle y Weber (2003) y por Luk (2003). Estos autores aplican dicho argumento solo a determinados segmentos de la población, ya que si el uso del automóvil se encuentra al alcance de las rentas altas, el transporte público es utilizado mayoritariamente por jóvenes y población de rentas bajas como única alternativa disponible.

Thevathasan y Balachandran (2007) argumentan que las razones que impulsan al uso del transporte público son de carácter personal y dependen de la percepción 
subjetiva del usuario, o de su experiencia negativa o positiva en el uso del mismo (Boisvert, 1998; Tyrinopoulos \& Antoniou, 2008). Andreassen (2005) considera que un factor clave de impulso para favorecer el uso del transporte público sería la aplicación de una tarifa por trayecto más reducida, unida a una velocidad comercial de desplazamiento más competitiva. En este sentido, la disponibilidad de un transporte urbano que garantice la movilidad de las personas de forma digna, oportuna, confiable y económica se constituye como una necesidad básica y prioritaria (Lupano \& Sánchez, 2009).

Estas premisas han configurado en todo momento las políticas, directrices y estrategias de las autoridades competentes en transporte y movilidad de la región de Madrid, aquellas tendientes a establecer un sistema de transporte público integrado, capaz de proporcionar un efectivo cambio modal que desplace de forma continuada y progresiva al automóvil, objetivo que el Consorcio Regional de Transportes de Madrid $(\mathrm{CRTM})^{3}$ ha desarrollado de forma decisiva. El CRTM ha conseguido articular un sistema altamente accesible en atención a las cuatro dimensiones que argumenta Hernández (2012), como son oferta (red ofrecida y su extensión), institucionalidad (estructura tarifaria y su regulación), rasgos individuales de los usuarios y sus ingresos y forma urbana (dinámica socioterritorial del transporte público). No obstante, dicho sistema de transporte, hasta la fecha, ha logrado únicamente un equilibrio entre el uso del automóvil y el transporte público (reparto modal). ${ }^{4}$ Esta situación, unida a la paralización de determinadas inversiones significativas por falta de recursos presupuestarios como consecuencia de la crisis, no es solo desfavorable a la posibilidad de solucionar el problema de congestión, sino que puede contribuir a empeorarlo.

Desde la perspectiva expuesta y a los efectos de solventar los problemas de congestión, cabe plantearse la siguiente cuestión: ¿cuál es la situación actual del transporte público en la región de Madrid para constituir una alternativa efectiva frente al automóvil ante la implantación de un peaje urbano? Según Muñoz, Simón y Jiménez (2014), la situación del transporte, con carácter general, es aceptable si consideramos que el transporte público en el centro urbano se caracteriza por una elevada accesibilidad y competitividad con respecto al automóvil, lo que permitiría asegurar un alto nivel de equidad en la movilidad y el transporte urbano. En cuanto al transporte público interurbano asociado a la movilidad radial, si bien se encuentra ciertamente desarrollado y adecuadamente interconectado al centro urbano a través de los intercambiadores de transporte que rodean el centro de la ciudad, puede dar lugar a cierta falta de equidad para aquellos usuarios con origen-destino en coronas metropolitanas más alejadas del centro urbano. Por último, respecto al transporte público transversal, cabe destacar que se encuentra escasamente desarrollado; no obstante, sería el escenario menos afectado por un peaje urbano, ya que sus usuarios

3 El CRTM, autoridad del transporte de la región, se creó en 1986, fecha desde la que viene desarrollando su actividad.

4 Según el informe sobre la Accesibilidad en el Transporte Público para la región de Madrid elaborado por el CRTM (http://www.crtm.es/media/157717/accesibilidad_transporte_publico_ madrid_folleto_bilingue.pdf, 2013), la distribución entre los modos privado/público es del $50 \% / 50 \%$. 
cuentan con mayores alternativas de movilidad mediante el vehículo propio, dada la elevada oferta existente de infraestructuras viales (carreteras de circunvalación). En resumen, el sistema de transporte público de la región de Madrid podría calificarse a priori de aceptable a la hora de garantizar un nivel de equidad social razonable ante la implantación de un peaje, si bien susceptible de incorporar determinadas estrategias que, asociadas al propio peaje, mejoren la equidad social para determinados grupos.

\section{Metodología, encuesta y cuestionario}

\section{Metodología aplicada}

Para la configuración del peaje urbano teórico propuesto para Madrid, y a los efectos de aplicar la encuesta poblacional de movilidad, se han considerado dos elementos clave, como son los objetivos prioritarios que el peaje debe satisfacer, y el diseño o esquema base para el cumplimiento de dichos objetivos prioritarios. ${ }^{5}$

Respecto a la identificación de los objetivos prioritarios (Muñoz, 2012, pp. 262-265), hemos efectuado, en primer lugar, un análisis de los objetivos generales y específicos contenidos en las estrategias de movilidad y transporte para la región de Madrid, con el fin de identificar las principales limitaciones que dichas medidas presentan, para, en segundo lugar, y sobre la base de los objetivos propios que persigue todo peaje urbano, identificar como objetivos prioritarios aquellos que presentan un mayor nivel de contribución a la superación de las limitaciones identificadas en dichas estrategias. En el caso de Madrid, a nuestro juicio, los objetivos que deben ser considerados como prioritarios son la búsqueda de mayor eficiencia en la movilidad y el transporte, la equidad social y la obtención de ingresos extraordinarios procedentes del sistema, considerando este último objetivo bajo la perspectiva de la redistribución social de dichos beneficios a favor de la movilidad sostenible. Por otro lado, el conjunto de objetivos inherentes a todo sistema de peaje, como son los vinculados a medioambiente, habitabilidad urbana y crecimiento económico, se han considerado como objetivos secundarios. Si bien contribuyen a una mejora de la sostenibilidad, no se han tenido en cuenta a los efectos de nuestra investigación, para simplificar el objeto de estudio.

En relación con la determinación del diseño o esquema teórico propuesto, han sido considerados cinco elementos clave que, de forma resumida, se exponen a continuación en la tabla 1 .

5 Las recomendaciones y conclusiones contenidas en el proyecto Curacao (www.isis-it.net/ curacao, 2009) determinan que el proceso de predicción sobre la viabilidad de un peaje urbano debe contemplar la definición de los objetivos que debe cumplir el sistema, el diseño o esquema teórico, la tecnología aplicable y el sistema de negocio para su gestión; estos dos últimos requisitos no han sido contemplados, por no ser considerados decisivos a los efectos de nuestro estudio. 
TABLA I Elementos básicos del diseño o esquema de peaje urbano propuesto

\begin{tabular}{|c|c|}
\hline ELEMENTO CLAVE & ALCANCE DEL ELEMENTO \\
\hline ¿A quién debe aplicarse la tarifa? & $\begin{array}{l}\text { Todo automóvil que acceda al centro urbano como sujeto potencial } \\
\text { de generación de congestión (principio de equidad). }\end{array}$ \\
\hline ¿Dónde debe ser aplicada? & $\begin{array}{l}\text { Primer cinturón orbital (M-30) como perímetro que inscribe y deli- } \\
\text { mita la zona objeto de protección por el peaje urbano. }\end{array}$ \\
\hline ¿Cuándo debe aplicarse la tarifa? & $\begin{array}{l}\text { Periodos de hora punta que en el caso de Madrid se producen entre } \\
7: 00 \text { y 10:00 horas y las 18:00 y 20:00 horas }\end{array}$ \\
\hline ¿Cuál debe ser la tarifa? & $\begin{array}{l}1,5 € \text { por acceso al centro urbano. Tarifa básica de aceptación equi- } \\
\text { valente a precio pagado por el transporte público en el interior de la } \\
\text { ciudad en el momento de la investigación. }\end{array}$ \\
\hline \multirow{3}{*}{$\begin{array}{l}\text { ¿Cómo deben emplearse los } \\
\text { fondos procedentes del sistema? }\end{array}$} & $\begin{array}{l}\text { Inversiones de primer orden: orientadas a garantizar la equidad hori- } \\
\text { zontal y vertical en la movilidad. }\end{array}$ \\
\hline & $\begin{array}{l}\text { Inversiones de segundo orden: orientadas a potenciar e impulsar } \\
\text { los modos más sostenibles sobre la base de infraestructura del peaje } \\
\text { urbano. }\end{array}$ \\
\hline & $\begin{array}{l}\text { Inversiones de tercer orden: orientadas a incrementas nuevas cuotas } \\
\text { de sostenibilidad en la movilidad y el transporte mediante inversiones } \\
\text { de carácter extraordinario. }\end{array}$ \\
\hline
\end{tabular}

FUENTE ELABORACIÓN PROPIA SEGÚN INFORMACIÓN CONTENIDA EN MUŃOZ (2012, PP. 283-285)

FIGURA I | Objetivos prioritarios del peaje urbano y su relación con el diseño o esquema teórico

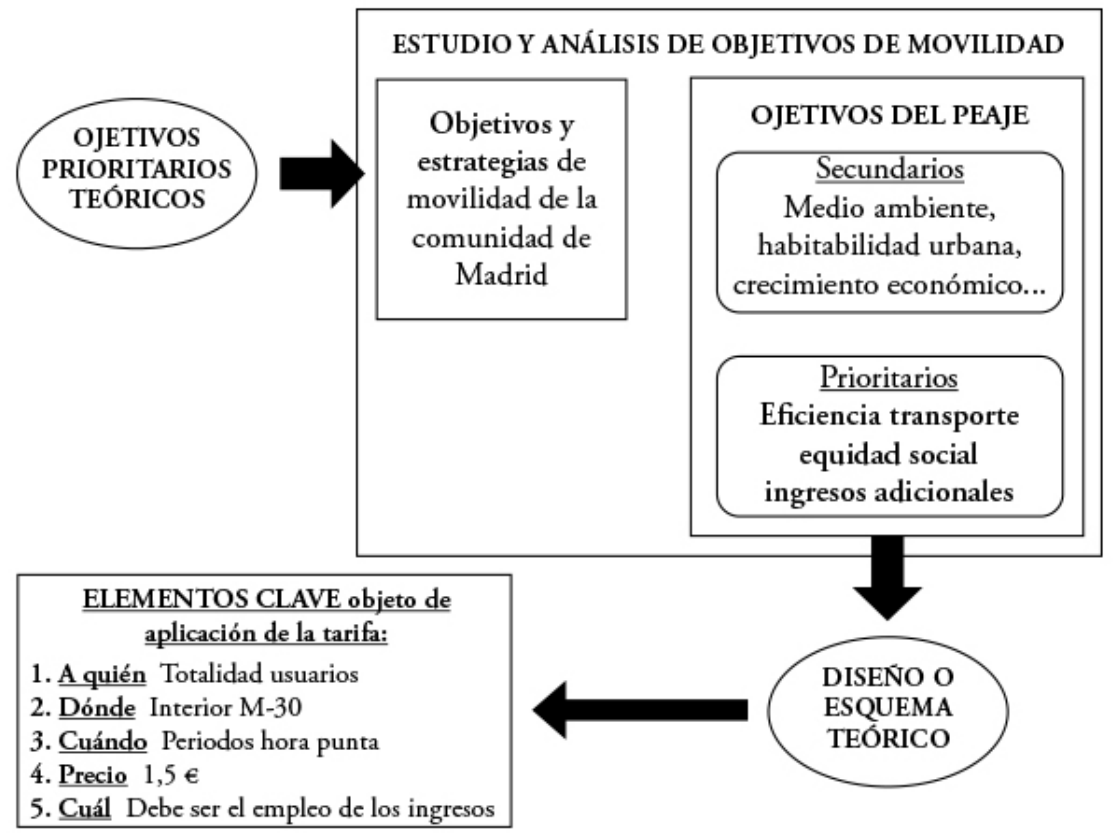

FUENTE ELABORACIÓN PROPIA 
La figura 1 refleja la interrelación entre los objetivos prioritarios asignados al sistema de peaje y el diseño o esquema teórico definido para el mismo.

Una vez configurado el sistema de peaje teórico en la forma propuesta en la figura 1, hemos de destacar que los aspectos metodológicos contemplados, los cuales ponen de manifiesto la complejidad del problema abordado, solo han sido considerados -a los efectos de la presente investigación- para determinar el cuestionario aplicado a la encuesta poblacional, con el objetivo de valorar y analizar el comportamiento del usuario de movilidad ante la implantación del peaje propuesto. Aspectos transcendentales, como el estudio y análisis de la equidad social, la aceptabilidad pública del sistema o las estrategias sobre inversiones asociadas al peaje propuesto, son objeto de líneas de investigación futura por nuestra parte.

Nuestra propuesta metodológica nos ha permitido identificar cualitativamente los cambios potenciales en las pautas de movilidad (impactos) como consecuencia de la aplicación de la tarifa de peaje sobre la población objetivo (usuarios de movilidad en la región de Madrid). Los impactos sobre la movilidad han sido definidos en función de los distintos flujos circulatorios existentes, de acuerdo con el concepto de utilidad en la movilidad que afecta a cada individuo ante el pago de la tarifa de peaje, el cual induce a adoptar una elección alternativa compensatoria que permita mantener o aumentar dicha utilidad en el transporte (Tversky, 1972). Los flujos circulatorios considerados se han identificado y asociado a tres escenarios de movilidad, según el origen y destino de desplazamientos:

i. Movilidad urbana: desplazamientos que tienen origen y destino en el interior de la ciudad de Madrid, los cuales no estarían sujetos al pago de la tarifa por encontrarse dentro de la zona protegida por el peaje.

ii. Movilidad radial: desplazamientos con origen-destino entre el centro urbano y las distintas coronas metropolitanas o viceversa. Se trata de usuarios que estarían obligados al pago de la tarifa en sus desplazamientos.

iii. Movilidad transversal: desplazamientos con origen-destino entre municipios pertenecientes a las distintas coronas metropolitanas. Se trata de usuarios que pueden verse afectados por el pago de la tarifa, pero que disponen de rutas alternativas para evitar el pago de la misma.

La figura 2 describe el área territorial de movilidad correspondiente a la región de Madrid; el teórico cordón de peaje propuesto coincidente con la vía de circunvalación M-30, cuyo perímetro es de aproximadamente $32,5 \mathrm{Km}$; y la tipología de flujos circulatorios objeto de estudio, según origen y destino de desplazamientos definidos anteriormente.

\section{Encuesta y cuestionario}

Para la determinación de la muestra, se ha considerado un universo poblacional constituido por 4.570 .990 usuarios sujetos a movilidad obligada. ${ }^{6}$ Dicho universo

Datos relativos a población activa en la Comunidad de Madrid representativa de usuarios sujetos a movilidad obligada por razón de trabajo, estudio o actividad cotidiana (Instituto Nacional de Estadística, 2012, www.ine.es). 
poblacional fue dividido en dos estratos, a efectos de selección de muestra: la población residente en el centro de la ciudad (2.205.147 usuarios) y los residentes en otros municipios de la Comunidad de Madrid (2.365.843 usuarios). Bajo la consideración estadística de población infinita, la ecuación $n=p(1-p) z_{\alpha}^{2} / \xi^{2}$ nos ha permitido determinar que la proporción de muestra representativa para cada estrato definido debe ser, al menos, de 600 usuarios, de acuerdo con un $\mathrm{p}=0.5$ como proporción esperada en el peor de los casos, dado que no se dispone de muestra piloto previa, y un nivel de significación asociado de $\alpha=0.05$ equivalente a un $95 \%$ de seguridad. El número de personas finalmente encuestadas, una vez concluido el proceso de recopilación de datos, se ha elevado a 630 usuarios con origen de desplazamientos en el centro de la ciudad y 668 usuarios con origen de desplazamientos en el resto de municipios de la Comunidad de Madrid. La tabla 2 a continuación recoge los resultados de la encuesta realizada.

Figura 2 Mapa de Madrid, cordón de peaje teórico y descripción de flujos de movilidad objeto de estudio

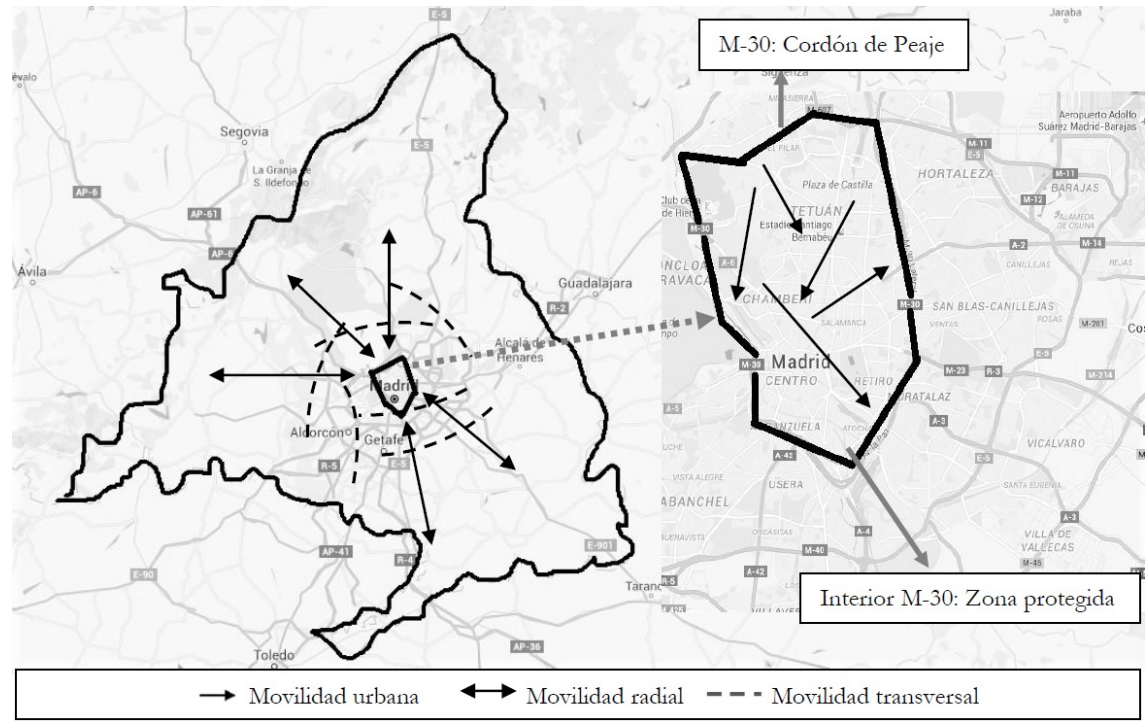

FUENTE HTTPS://WWW.GOOGLE.ES/MAPS Y ELABORACIÓN PROPIA. MAPA DE MADRID ADAPTADO A LOS OBJETIVOS DE LA INVESTIGACIÓN

TABLA 2 Usuarios encuestados y resultados de la encuesta

\begin{tabular}{|l|c|c|}
\cline { 2 - 3 } \multicolumn{1}{c|}{} & $\begin{array}{c}\mathbf{N}^{\mathbf{0}} \\
\text { ENCUESTADOS }\end{array}$ & $\%$ \\
\hline Total usuarios de movilidad & 1.298 & 100 \\
\hline Origen de desplazamiento & 630 & 49 \\
\hline Centro urbano & 668 & 51 \\
\hline Resto de municipios & \multicolumn{2}{|c|}{} \\
\hline
\end{tabular}




\begin{tabular}{|c|c|c|}
\hline & \multicolumn{2}{|c|}{ (continuación) } \\
\hline & $\begin{array}{c}\mathrm{N}^{\mathbf{0}} \\
\text { ENCUESTADOS }\end{array}$ & $\%$ \\
\hline \multicolumn{3}{|l|}{ Destino de desplazamiento } \\
\hline Centro urbano & 581 & 45 \\
\hline Resto de municipios & 717 & 55 \\
\hline \multicolumn{3}{|l|}{ Modo de transporte urbano utilizado habitualmente } \\
\hline Automóvil & 576 & 45 \\
\hline Transporte público & 679 & 52 \\
\hline Otros modos más sostenibles (bicicleta o caminar) & 43 & 3 \\
\hline \multicolumn{3}{|l|}{ Modo de transporte urbano disponible } \\
\hline Automóvil, transporte público y otros modos (bicicleta o caminar) & 319 & 25 \\
\hline Automóvil y transporte público & 551 & 42 \\
\hline Transporte público y otros modos (bicicleta o caminar) & 119 & 9 \\
\hline Solo transporte público & 280 & 22 \\
\hline Solo automóvil & 29 & 2 \\
\hline \multicolumn{3}{|l|}{ Modo de transporte urbano utilizado en caso de peaje urbano } \\
\hline Automóvil & 262 & 20 \\
\hline Automóvil fuera del horario de funcionamiento del peaje & 124 & 10 \\
\hline Automóvil hasta el acceso al peaje y cambia a transporte público & 35 & 3 \\
\hline Automóvil mediante cambio a otra ruta alternativa & 38 & 3 \\
\hline Transporte público & 775 & 59 \\
\hline Otros modos más sostenibles (bicicleta o caminar) & 64 & 5 \\
\hline
\end{tabular}

FUENTE ELABORACIÓN PROPIA SEGÚN DATOS DE “ENCUESTA SOBRE MOVILIDAD URBANA COMUNIDAD DE MADRID”

\section{Impactos de movilidad derivados del peaje urbano}

La información recopilada a través de la encuesta y clasificada de acuerdo con los tres escenarios objeto de estudio y análisis, tal como se muestra en la tabla 3, permite identificar el uso de los modos de transporte antes y después de la implantación del peaje urbano propuesto, así como el cambio en el comportamiento de movilidad adoptado, lo que aporta los datos necesarios para deducir los impactos sobre la movilidad urbana como consecuencia de dicha medida.

TABLA 3 Comportamiento de movilidad antes y después de peaje urbano por cada escenario de movilidad

\begin{tabular}{|l|c|c|c|c|c|c|c|c|c|}
\cline { 2 - 9 } \multicolumn{1}{c|}{} & \multicolumn{7}{c|}{ ESCENARIOS DE MOVILIDAD } \\
\cline { 2 - 10 } \multicolumn{1}{c|}{} & URBANA & $\%$ & RADIAL & $\%$ & $\begin{array}{c}\text { TRANS- } \\
\text { VERSAL }\end{array}$ & $\%$ & TOTAL & $\%$ \\
\hline Total usuarios & 411 & $100 \%$ & 389 & $100 \%$ & 498 & $100 \%$ & 1.298 & $100 \%$ \\
\hline Modos de transporte antes del peaje urbano \\
\hline Automóvil & 180 & $44 \%$ & 189 & $49 \%$ & 207 & $42 \%$ & 576 & $45 \%$ \\
\hline
\end{tabular}

(continúa) 
(continuación)

\begin{tabular}{|c|c|c|c|c|c|c|c|c|}
\hline & & & & & & & & \\
\hline & \multicolumn{8}{|c|}{ ESCENARIOS DE MOVILIDAD } \\
\hline & URBANA & $\%$ & RADIAL & $\%$ & $\begin{array}{l}\text { TRANS- } \\
\text { VERSAL }\end{array}$ & $\%$ & TOTAL & $\%$ \\
\hline Transporte público & 209 & $51 \%$ & 200 & $51 \%$ & 270 & $54 \%$ & 679 & $52 \%$ \\
\hline $\begin{array}{l}\text { Otros modos más sostenibles } \\
\text { (bicicleta o caminar) }\end{array}$ & 22 & $5 \%$ & 0 & $0 \%$ & 21 & $4 \%$ & 43 & $3 \%$ \\
\hline \multicolumn{9}{|c|}{ Modos de transporte después del peaje urbano } \\
\hline Automóvil & 95 & $24 \%$ & 158 & $41 \%$ & 206 & $41 \%$ & 459 & $35 \%$ \\
\hline Transporte público & 273 & $66 \%$ & 231 & $59 \%$ & 271 & $55 \%$ & 775 & $60 \%$ \\
\hline $\begin{array}{l}\text { Otros modos más sostenibles } \\
\text { (bicicleta o caminar) }\end{array}$ & 43 & $10 \%$ & 0 & $0 \%$ & 21 & $4 \%$ & 64 & $5 \%$ \\
\hline \multicolumn{9}{|c|}{ Comportamiento de movilidad en peaje urbano } \\
\hline $\begin{array}{l}\text { Continua con automóvil y } \\
\text { paga la tarifa de peaje }\end{array}$ & 88 & $21 \%$ & 53 & $14 \%$ & 40 & $8 \%$ & 181 & $14 \%$ \\
\hline $\begin{array}{l}\text { Continua con transporte } \\
\text { público }\end{array}$ & 189 & $46 \%$ & 182 & $47 \%$ & 244 & $49 \%$ & 615 & $47 \%$ \\
\hline $\begin{array}{l}\text { Continua con otros modos } \\
\text { más sostenibles (bicicleta o } \\
\text { caminar) }\end{array}$ & 19 & $5 \%$ & 0 & $0 \%$ & 21 & $4 \%$ & 40 & $3 \%$ \\
\hline $\begin{array}{l}\text { Cambia a automóvil y paga la } \\
\text { tarifa de peaje }\end{array}$ & 7 & $2 \%$ & 5 & $1 \%$ & 4 & $1 \%$ & 16 & $1 \%$ \\
\hline Cambia a transporte público & 84 & $20 \%$ & 49 & $13 \%$ & 27 & $5 \%$ & 160 & $12 \%$ \\
\hline $\begin{array}{l}\text { Cambia a otros modos más } \\
\text { sostenibles }\end{array}$ & 24 & $6 \%$ & 0 & $0 \%$ & 0 & $0 \%$ & 24 & $2 \%$ \\
\hline $\begin{array}{l}\text { Anticipa o retrasa } \\
\text { desplazamiento para evitar el } \\
\text { pago de la tarifa }\end{array}$ & 0 & $0 \%$ & 84 & $21 \%$ & 40 & $8 \%$ & 124 & $10 \%$ \\
\hline $\begin{array}{l}\text { Automóvil hasta el acceso al } \\
\text { peaje y cambia a transporte } \\
\text { público }\end{array}$ & 0 & $0 \%$ & 16 & $4 \%$ & 19 & $4 \%$ & 35 & $3 \%$ \\
\hline $\begin{array}{l}\text { Utiliza el automóvil mediante } \\
\text { cambio a una ruta alternativa } \\
\text { para evitar el pago de la tarifa }\end{array}$ & 0 & $0 \%$ & 0 & $0 \%$ & 38 & $8 \%$ & 38 & $3 \%$ \\
\hline $\begin{array}{l}\text { Automóvil por no verse } \\
\text { afectados }\end{array}$ & 0 & $0 \%$ & 0 & $0 \%$ & 65 & $13 \%$ & 65 & $5 \%$ \\
\hline
\end{tabular}

FUENTE ELABORACIÓN PROPIA SEGÚN DATOS DE "ENCUESTA SOBRE MOVILIDAD URBANA COMUNIDAD DE MADRID"

\section{Impacto sobre el reparto modal en modos de transporte}

El impacto global en el reparto modal tras la implantación del peaje urbano situaría el uso del automóvil en un $35 \%$ frente al $45 \%$ preexistente, tal y como refleja la figura 3. Este diferencial de 10 puntos se traduce en un aumento a favor del uso de modos de transporte sostenibles, como son un incremento de 8 puntos a favor del transporte público y 2 puntos a favor del uso de la bicicleta o caminar, atribuible en este último caso al centro de la ciudad.

La mayor contribución en el cambio modal hacia modos más sostenibles se produce en el centro de la ciudad, que experimenta una reducción en el uso del automóvil de 20 puntos, con una transferencia de 15 puntos a favor del transporte público urbano debido a su alta competitividad y accesibilidad, y 5 puntos a favor de otros modos más sostenibles, como son el uso de la bicicleta o caminar, debido 
a la existencia de determinadas infraestructuras viales para el desarrollo de este tipo de movilidad en el centro urbano.

El cambio en el reparto modal atribuible a la movilidad radial es moderado, de 8 puntos a favor íntegramente del transporte público interurbano, fundamentalmente debido a su menor competitividad con respecto al automóvil. Por su parte, el cambio atribuible a la movilidad transversal es insignificante, debido al escaso desarrollo del transporte público en este tipo de desplazamientos, donde, además, el elevado desarrollo de infraestructuras viales hace que el automóvil sea el modo más competitivo en cuanto a tiempos de desplazamiento, e incluso el único modo de transporte disponible para aquellas zonas más alejadas del centro urbano.

FIGURA 3 | Distribución del reparto modal antes y después de la implantación del peaje urbano

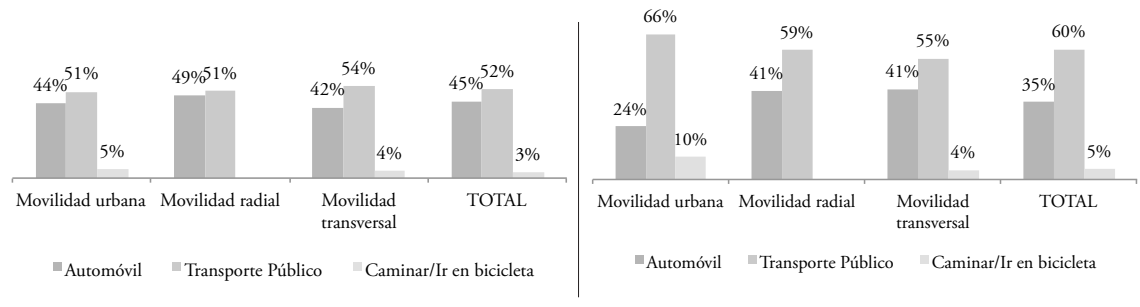

FUENTE ELABORACIÓN PROPIA

Reducción en el uso del automóvil vs. incremento en el uso de modos más sostenibles

La reducción en el uso del automóvil como consecuencia del peaje, tal como refleja la figura 4 , alcanza un $20 \%$, que se traduce en un aumento del transporte público del $14 \%$ y del $49 \%$ en el uso de la bicicleta o caminar.

FIGURA 4 Reducción del uso del automóvil y transferencia hacia el uso de modos más sostenibles

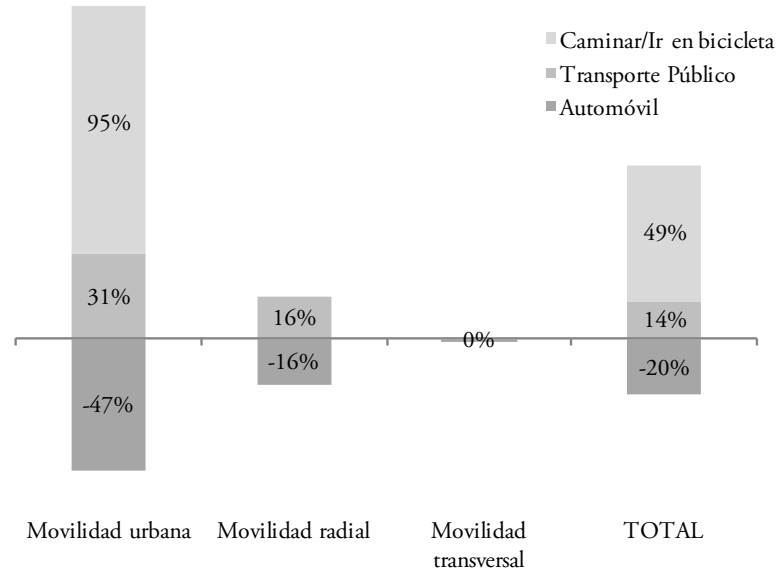

FUENTE ELABORACIÓN PROPIA 
La mayor contribución en la reducción del automóvil es aportada por el centro urbano, con un $47 \%$, hecho relevante si consideramos que estos desplazamientos, por tener origen-destino en el interior de la ciudad, no se verían afectados por el pago de la tarifa. Esta situación se debe, fundamentalmente, a las mejores condiciones de movilidad (menor intensidad del tráfico) y de habitabilidad urbana que impondría el peaje. Al aumentar su velocidad de circulación, el transporte público urbano en superficie gana mayor competitividad, razón por la que este modo experimentaría un incremento del $31 \%$. El uso de modos como la bicicleta o caminar experimentaría un aumento del $95 \%$, debido al mejor uso de las infraestructuras asociadas a ellos ante una menor intensidad de tráfico en la zona.

La reducción del uso del automóvil aportada por la movilidad radial en los desplazamientos que discurren entre el centro de la ciudad y la periferia, o viceversa, es del $16 \%$, reducción más moderada que el escenario anterior, que se traduce íntegramente en una transferencia hacia el transporte público interurbano. El hecho de que dichos desplazamientos se vean afectados de forma directa por el pago de tarifa, unido a la existencia de un transporte público interurbano menos competitivo con respecto al automóvil, pone de manifiesto un riesgo potencial de cierta injusticia o inequidad en la movilidad impuesta por el sistema. Ello afectaría fundamentalmente a aquellos conductores situados en municipios pertenecientes a las coronas metropolitanas más alejadas del centro urbano, con elevado grado de cautividad por su dependencia del automóvil. No obstante, esta regresividad puede suponer un cierto beneficio para los usuarios del transporte público que utilizan este modo por disponer de menos ingresos, ya que se enfrentarían a una menor congestión y, además, facilitaría una mayor tasa de ocupación de dicho modo (De Grange \& Troncoso, 2015).

La reducción en el uso del automóvil para el escenario de movilidad transversal relativa a los desplazamientos interperiféricos es prácticamente nula. Este constituye un impacto perfectamente predecible, ya que se trata de desplazamientos intermunicipales que no se verían afectados en la mayoría de los casos por el peaje, debido a que el automóvil se muestra más competitivo, al ser escaso el desarrollo y la accesibilidad del transporte público para este escenario.

\section{La reducción de los niveles de congestión}

La reducción de la congestión ha sido cuantificada en términos de número de automóviles circulando durante el horario de funcionamiento del peaje que, en nuestro caso, hace referencia a los periodos horarios de máxima concentración de congestión comprendidos entre las 07:00 y las 10:00 horas y las 18:00 y 20:00 horas.

La figura 5 revela que se produce una reducción muy significativa, del orden del $55 \%$, durante los periodos de funcionamiento del peaje. Esta importante reducción de congestión pone de manifiesto dos aspectos relevantes: en primer lugar, la gran eficacia del peaje para desincentivar el uso del automóvil durante los horarios tarificados; y en segundo lugar, la alta sensibilidad del usuario al pago de una tarifa como la aplicada en nuestro estudio, de $1,5 €$ por acceso, tarifa básica y aceptable si la comparamos con las $10,5 £$ por acceso aplicables en el peaje londinense (TfL Congestion Charge, 2015). 
El comportamiento de los usuarios que continúan usando el automóvil pone de manifiesto que en un 16\% utilizarían el automóvil en horario tarificado y estarían exentos del pago de tarifa por tratarse de usuarios residentes en el centro urbano, cuyos desplazamientos tienen origen y destino dentro del cordón de peaje; un $11 \%$ correspondería a usuarios que no se verían afectados por el peaje; y un $18 \%$ de usuarios asumiría el pago de la tarifa de peaje y continuaría utilizando el automóvil.

\section{FIGURA 5 $\mid$ Reducción de la congestión en horario de funcionamiento del peaje urbano}

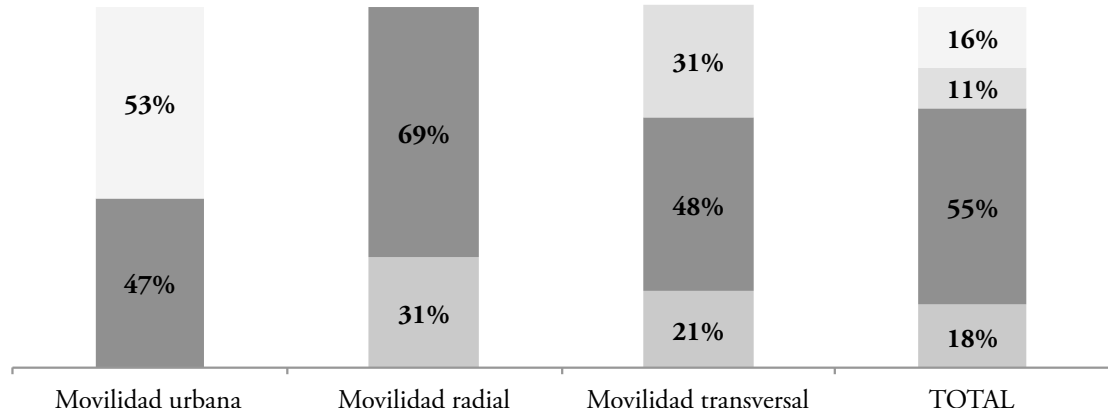

Pagan tarifa de peaje

No afectados por tarifa de peaje
- No utilizan automóvil en horario de peaje

Vehículos exentos de peaje

FUENTE ELABORACIÓN PROPIA

Un análisis detallado permite apreciar de forma más completa la contribución a la reducción de la congestión por cada escenario estudiado.

En primer lugar, respecto al escenario de movilidad urbana como zona objeto de reducción de congestión, se observa que un $47 \%$ de los usuarios abandona el uso del automóvil para realizar sus desplazamientos mediante transporte público urbano, o bien mediante bicicleta o caminar, lo que constituye una reducción de congestión efectiva y definitiva. Este significativo indicador -a pesar de que dichos usuarios no se verían afectados por el pago de la tarifa- se debe a la elevada accesibilidad del transporte público en la zona, cuya velocidad comercial se vería mejorada por la reducción de congestión en el interior de la ciudad. Tal circunstancia reafirma nuestra hipótesis de que un transporte público adecuado y accesible es condición necesaria como alternativa al automóvil ante la imposición de una tarifa de peaje; en este caso concreto, dicho argumento queda reforzado por el hecho de que los usuarios de ese tipo no estarían sujetos al pago de la tarifa, y su decisión de cambiar al transporte público se produce como consecuencia de un comportamiento más sostenible en la movilidad.

No obstante lo anterior, el elevado indicador del 53\% de usuarios que continuarían utilizando el automóvil en desplazamientos internos no se muestra coherente con la alta disponibilidad de transporte público en la zona. Este indicador, y su 
evolución, deberán ser objeto de especial atención a los efectos de imponer medidas complementarias; incluso, en casos extremos, podría hacer necesaria la imposición de medidas coercitivas para garantizar la equidad con respecto al resto de usuarios que se encuentran obligados al pago de la tarifa por acceder al centro urbano.

En segundo lugar, el escenario de movilidad radial muestra una reducción del tráfico circulatorio del $69 \%$ durante el horario sujeto a tarificación, indicador igualmente significativo, si bien tan solo un 32\% debe considerarse reducción efectiva por cambio al uso del transporte público. Tal cambio es fundamentalmente atribuible a usuarios que residen en las coronas metropolitanas más próximas al centro de la ciudad, donde el desplazamiento radial, mediante transporte público interurbano, ofrece mejores oportunidades. El 37\% restante de usuarios contribuye a una reducción de congestión que se desplaza hacia horarios no tarificados (congestión desplazada).

Por último, la situación en el escenario de movilidad transversal revela que la reducción de la congestión durante el periodo de funcionamiento del sistema de peaje se sitúa en el $48 \%$. No obstante, de forma análoga a lo expuesto para el escenario anterior, la reducción efectiva es de un $21 \%$ por cambio al uso del transporte público interurbano, mientras que el $27 \%$ restante es congestión trasladada a horarios no tarificados.

Un examen de la congestión efectiva y la congestión desplazada, de acuerdo con los indicadores que se exponen en la figura 6, muestra que un 60\% de los usuarios abandona el uso del automóvil durante el horario de funcionamiento del peaje, para utilizar el transporte público, la bicicleta o caminar en sus desplazamientos, lo que permite hablar de congestión efectiva o permanente. Por su parte, el $40 \%$ restante ha dejado de utilizar el automóvil durante el horario de funcionamiento, pero traslada sus desplazamientos hacia horarios no tarificados, anticipando o retrasando el momento de realizar sus viajes, o bien cambiando de ruta para evitar el pago de la tarifa. Este relevante indicador de congestión desplazada en la movilidad radial y transversal es altamente probable en Madrid, dado el progresivo incremento de la flexibilidad horaria laboral y la elevada oferta vial orbital existente, situación que daría lugar a que zonas y periodos horarios no tarificados que no presentan problemas de congestión comiencen a saturarse, generando un potencial efecto borde o barrera en las inmediaciones del cordón de peaje. No obstante, ello presenta la ventaja de disminuir la congestión circulatoria durante los intervalos de hora punta y en la zona vial donde opera el peaje. En cualquier caso, este impacto debe ser considerado a los efectos de incorporar determinadas estrategias complementarias para su control.

El análisis por escenarios de movilidad indica que, en el centro de la ciudad, la reducción de la congestión debe considerarse íntegramente como efectiva o permanente por cambio modal al transporte público urbano. Los escenarios de movilidad radial y movilidad transversal revelan indicadores muy similares, con una reducción de la congestión efectiva del $46 \%$ y $43 \%$, respectivamente, por cambio al uso de modos sostenibles, si bien la congestión desplazada hacia horarios no tarificados se eleva al 54\% y 57\%, respectivamente. Estos significativos indicadores de congestión desplazada generarían riesgo potencial de efecto borde o barrera, como hemos afirmado en el párrafo anterior. Cabría destacar que la implantación de una red estratégica de aparcamientos disuasorios que permitan intensificar la alternativa de 
intermodalidad combinada de automóvil y transporte público en los límites del acceso al cordón de peaje, podría suponer una solución factible para reducir dicha congestión desplazada, al mismo tiempo que se disminuye la congestión efectiva en el interior del cordón.

FIGURA 6 | Reducción de congestión efectiva y congestión desplazada hacia horarios no tarificados

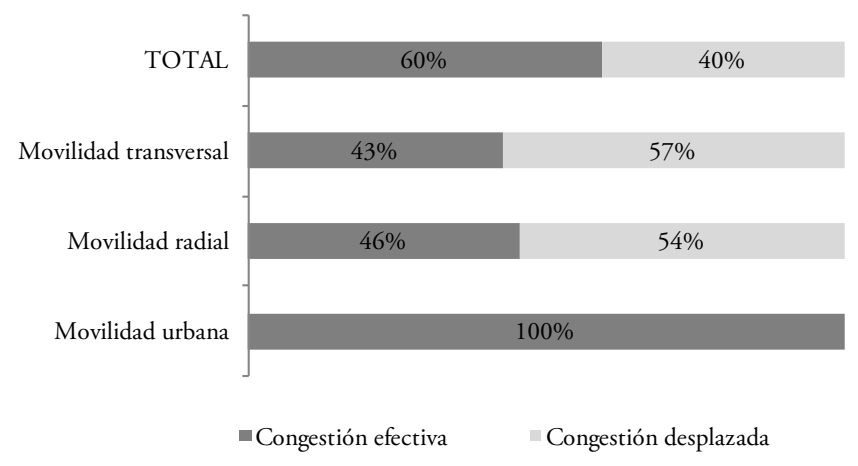

FUENTE ELABORACIÓN PROPIA

\section{El grado de permanencia en modos sostenibles}

El alto nivel de permanencia en el uso de modos de transporte sostenible, como se refleja en la figura 7, demuestra el escaso riesgo, a priori, del peaje para impulsar el denominado 'efecto llamada o inducción al uso del automóvil' como consecuencia de la percepción, por parte de los usuarios, de una mejora potencial de los tiempos de desplazamiento en sus viajes ante el menor nivel de congestión que aportaría el sistema. Tan solo un 2\%, por término medio, abandonaría el uso del transporte público para realizar sus desplazamientos mediante automóvil.

FIGURA 7 Grado de permanencia en el uso de modos sostenibles tras la implantación del peaje urbano

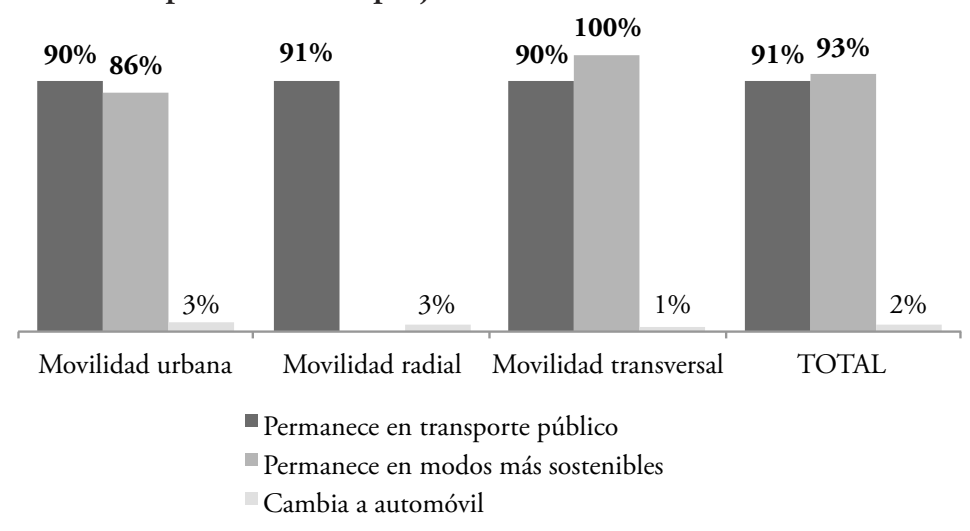

FUENTE ELABORACIÓN PROPIA 


\section{Conclusiones}

Los principales impactos derivados de la implantación de un peaje urbano para Madrid, como el propuesto, ponen de manifiesto que dicha medida, con carácter general, tendría un efecto altamente positivo sobre la mejora de la sostenibilidad de la movilidad urbana en el centro de la ciudad y su entorno. Tal efecto obedecería principalmente a la elevada capacidad del peaje para impulsar un efectivo cambio modal a favor del uso de modos más sostenibles, como son el transporte público tanto urbano como interurbano, y otros modos también más sostenibles, como el uso de la bicicleta o caminar en el centro de la ciudad. El sistema de peaje proporcionaría una reducción significativa del $20 \%$ en el uso del automóvil, cifra a priori muy similar a los resultados que peajes como el londinense o el peaje de Estocolmo ofrecían tras su puesta en funcionamiento (18\% y $25 \%$, respectivamente). El impulso que el peaje proporciona al uso de los modos de transporte sostenible aludidos anteriormente es relevante, si consideramos que, hasta la fecha y fruto de la intensa política de fomento y desarrollo del transporte urbano llevada a cabo por las autoridades competentes desde el año 1986, tan solo se ha conseguido alcanzar un cierto equilibrio en el reparto modal entre automóvil y transporte público. No obstante, este cambio modal cuantitativo a favor de los modos más sostenibles requiere un análisis cualitativo profundo, que permita valorar si las condiciones de movilidad de los usuarios que cambian a dicho modo se ven alteradas o no y en qué medida.

La reducción en el uso del automóvil es muy significativa en el centro de la ciudad, debido a que un elevado número de usuarios optaría por cambiar a los modos de transporte más sostenibles, ante la menor congestión y mejor habitabilidad urbana que proporcionaría el sistema. Una reducción más moderada en el uso del automóvil, pero también destacada, se produce en el escenario de movilidad radial.

El sistema de peaje muestra una alta capacidad para reducir de forma muy significativa la congestión durante el horario de funcionamiento, un proceso que puede considerarse en gran medida como definitivo, debido al cambio modal a favor del transporte público que provoca el sistema. Un nivel importante de la reducción del tráfico durante el horario de peaje se debe a congestión que se traslada hacia horarios no tarificados, fundamentalmente ocasionada por usuarios de movilidad radial y transversal que disponen de flexibilidad horaria en sus desplazamientos. Este tipo de congestión desplazada tendría finalmente incidencia directa en la congestión del centro urbano como zona protegida, y denota cierta ineficacia del peaje. No obstante, tiene el efecto positivo de reducir el tráfico circulatorio en los periodos de hora punta y trasladarlo hacia horarios menos saturados de congestión; y el efecto negativo de provocar efecto barrera en las inmediaciones del cordón, problema que pudiera ser controlado bien mediante ampliaciones de los intervalos sujetos a tarificación según niveles de tráfico o, en el caso más extremo, mediante la aplicación de un horario de tarificación continuado similar al peaje londinense.

Como conclusión final, podemos afirmar que la aplicación del peaje propuesto para Madrid presenta, a priori, un alto grado de viabilidad y capacidad para desincentivar el uso del automóvil, dado el elevado nivel de tráfico circulatorio cada vez más acuciante y estructural. Dicha medida permitiría un desarrollo más sostenible e 
integral del transporte urbano, induciendo a la potenciación del uso del transporte público como pilar básico de las políticas y objetivos de movilidad que persiguen las autoridades de transporte en la Comunidad de Madrid. Sin embargo, y coincidiendo con el argumento que plantean Gibson y Carnovale (2015), hemos de ser conscientes de que la efectividad del peaje para reducir la congestión depende de cómo los conductores responden frente a la medida y de cuál es el alcance de su respuesta, por lo que un fuerte apoyo institucional a través de campañas de concienciación y participación ciudadana constituye una pieza clave para reducir el problema de la aceptación social.

A modo de reflexión, destacamos una serie de consideraciones relevantes que intensificarían la utilización del transporte público frente al automóvil. Entre ellas: (i) la creación de carriles Bus- $\mathrm{Vao}^{7}$ en las vías principales que comunican la periferia con el centro urbano, los cuales permitirían mejorar la competitividad del transporte público interurbano y estimular su uso, así como el de coche compartido; (ii) a construcción de una red de aparcamientos disuasorios próximos a los principales intercambiadores que rodean la ciudad, que intensificaría la intermodalidad combinada de vehículo propio y transporte público urbano en los límites del acceso al cordón y reduciría el uso del automóvil en el centro urbano; y (iii), por último, la potenciación del transporte ferroviario radial de cercanía como modo de alta ocupación, que reduciría notablemente el uso del automóvil en los desplazamientos radiales, mejorando la competitividad, frecuencia e interconexión con el centro de la ciudad.

Por último, la aceptabilidad social, el estudio y análisis exhaustivo de las implicaciones del peaje sobre la equidad en la movilidad o la aplicación de estrategias vinculadas al peaje y desarrolladas mediante inversiones económicamente rentables y sostenibles y su financiación, constituyen líneas de investigación futuras abiertas sobre la base del presente artículo, que darán lugar a nuevos trabajos por nuestra parte.

\section{Referencias bibliográficas}

Albalate, D. \& Bel, G. (2009). What local policy makers should know about urban road charging: Lessons from worldwide experience. Public Administration Review, 69(5), 962-975. https://dx.doi.org/10.1111/j.1540-6210.2009.02045.x

Anas, A. \& Lindsey, R. (2011). Reducing urban road transportation externalities: Road pricing in theory and in practice. Review of Environmental Economics and Policy, 5(1), 66-88. https://dx.doi.org/10.1093/reep/req019

Andreassen, T. (2005). (Dis)satisfaction with public service: The case of public transportation. Journal of Service Marketing, 9(5), 30-41. https://dx.doi. org/10.1108/08876049510100290

7 Los carriles vaO deben su nombre a que son carriles para Vehículos con Alta Ocupación. Se fija un número mínimo de ocupantes, se señaliza el carril explicando cuántas personas deben viajar a bordo del vehículo para poder emplear el carril y quien lo cumple puede marchar por el carril vaO, que es más ágil que los demás. Véase Circula Seguro. Tu publicación sobre seguridad vial, en http:// www.circulaseguro.com/que-es-un-carril-vao/ [N. de E.] 
Bamberg, S., Rölle, D. \& Weber, C. (2003). Does habitual car use not lead to more resistance to change of travel mode? Transportation, 30(1), 97-108. http://dx.doi. org.10.1023/A:1021282523910

Banister, D. (2003). Critical pragmatism and congestion charging in London. International Social Science Journal, 55(176), 249-264. https://dx.doi.org.10.1111/j.14682451.2003.05502006.x

Bayona, J. \& Pujadas, I. (2014). Movilidad residencial y redistribución de la población metropolitana: los casos de Madrid y Barcelona. EURE, 40(85), 281-287. http:// dx.doi.org/10.4067/S0250-71612014000100012

Boisvert, D. (1998). Switching to public transport. 22 $2^{\text {nd }}$ Australasian Transport Research Forum (pp. 649-666). En http://www.atrf.info/papers/1998/1998_Boisvert.pdf

Bonsall, P. \& Kelly, C. (2005). Road user charging and social exclusion: The impact of congestion charges on at risk groups. Transport Policy, 12(5), 406-418. https://doi. org/10.1016/j.tranpol.2005.06.007

Consorcio Regional de Transportes de Madrid (CRTM). (2013). Accesibilidad en el transporte público de Madrid. En http://www.crtm.es/media/157717/accesibilidad_transporte_ publico_madrid_folleto_bilingue.pdf

Curacao (2009a). Deliverable D2: State of the Art Report, Curacao. Project Sponsored by European Commission under the FPC Framework.

Curacao (2009b). Deliverable D3: Case Study results, Curacao. Project funded by the European Commission under the FP6 Framework.

De Grange, L. \& Troncoso, R. (2015). Límites de la tarificación vial. EURE, 41(122), 167-184. http://dx.doi.org/10.4067/S0250-71612015000100008

Eliasson, J. (2008). Lessons from the Stockholm congestion charging trial. Transport Policy, 15(6), 395-404. https://doi.org/10.1016/j.tranpol.2008.12.004

Eliasson, J., Hultkrantz, L., Nerhagen, L. \& Rosqvist, L. S. (2009). The Stockholm congestioncharging trial 2006: Overview of effects. Transportation Research Part A: Policy and Practice, 43(3), 240-250. https://doi.org/10.1016/j.tra.2008.09.007

Eriksson, L., Garvill, J. \& Nordlund, A. (2006). Acceptability of travel demand management measures: The importance of problem awareness, personal norm, freedom, and fairness. Journal of Environmental Psychology, 26(1), 15-26. https://doi.org/10.1016/j. jenvp.2006.05.003

Fundación RACC (2009). La congestión en los corredores de acceso a Madrid. Enero 2009. En http://imagenes.racc.es/pub/ficheros/adjuntos/adjuntos_estudio_congestion_webb_ jzq_9adadbd7.pdf

Gibson, M. \& Carnovale, M. (2015) .The effects of road pricing on driver behavior and air pollution. Journal of Urban Economics, 89, 62-73. https://doi.org/10.1016/j. jue.2015.06.005

Giuliano, G. (1994). Equity and fairness considerations of congestion pricing. En Curbing Gridlock: Peak-Period Fees to Relieve Traffic Congestion. Volume Two: Commissioned Papers (pp. 250-279). Washington, DC: National Research Council. En http://bit. $\mathrm{ly} / 2 \mathrm{rXkoHm}$

Hensher, D. A. \& Prioni, P. (2002). A service quality index for area-wide contract performance assessment. Journal of Transport Economics and Policy, 36(1), 93-113. En http://bit. ly/2rX8c9l 
Hernández, D. (2012). Activos y estructuras de oportunidades de movilidad. Una propuesta analítica para el estudio de la accesibilidad por transporte público, el bienestar y la equidad. EURE, 38(115), 117-135. http://dx.doi.org/10.4067/S025071612012000300006

Holland E. P. \& Watson P. L. (1978). Traffic restraint in Singapore 1: Measuring the effects of the area licence scheme. Traffic Engineering and Control, 19(1), 14-22.

InstitutoNacional de Estadística (INE), España. (2012). Estadísticas sobre población activa en la Comunidad de Madrid. En http://www.ine.es

Jaensirisak, S., Wardman, M. \& May, A. D. (2005). Explaining variations in public acceptability of road pricing schemes. Journal of Transport Economics and Policy, 39(2), 127-153. En http://eprints.whiterose.ac.uk/2430/1/ITs_Explaining_variations_uploadable.pdf

Leape, J. (2006). The London congestion charge. Journal of Economic Perspectives, 20(4), 157 176. http://doi.10.1257/jep.20.4.157

Luk, J. (2003). Reducing car travel in Australian cities: Review report. Journal of Urban Planning and Development, 129(2), 84-96.

Lupano, J. A. \& Sánchez, R. J. (2009). Politicas de movilidad urbana e infraestructura urbana de transporte. Documento de proyecto [LC/W.230]. Santiago, Chile: Comisión Económica para América Latina y el Caribe (CEPAL) / France Cooperation. En http:// repositorio.cepal.org/bitstream/handle/11362/3642/1/S2009021_es.pdf

Mogridge, M. (1986). Road pricing: The right solution for the right problem? Transportation Research A, 20(2), 157-167. https://doi.org/10.1016/0191-2607(86)90043-9

Mogridge, M. \& Holden, D. (1987). A panacea for road congestion?: A riposte. Traffic Engineering \& Control, 28(1), 13-19.

Muñoz, J. P. (2012). Los sistemas de peaje urbano: estudio y predicción de los efectos derivados de la implantación de un sistema de peaje urbano para la Ciudad de Madrid. Tesis Doctoral, Universidad Rey Juan Carlos, Madrid, España.

Muñoz, J. P., Simón, C. \& Jiménez, I. C. (2014). Estudio empírico sobre la utilización del transporte público en la Comunidad de Madrid como factor clave de movilidad sostenible. Cuadernos de Economía - Spanish Journal of Economics and Finance, 104(37), 112-124. https://doi.org/10.1016/j.cesjef.2013.12.001

Pigou, A.C. (1920). The economics of welfare, Londres: MacMillan.

Pozueta, J. (2008). La experiencia internacional en peajes urbanos. Cuadernos de Investigación Urbanistica, (58), 3-112. En http://polired.upm.es/index.php/ciur/article/view/273/268

Rotaris, L., Danielis, R., Marcucci, E. \& Massiani, J. (2010). The urban road pricing scheme to curb pollution in Milan, Italy: description, impacts and preliminary cost-benefit analysis assessment. Transportation Research Part A, 44(5), 359-375. https://doi. org/10.1016/j.tra.2010.03.008

Santos, G. \& Fraser, G. (2006). Road pricing: Lessons from London. Economic Policy, 21(46), 264-310. https://doi.org/10.1111/j.1468-0327.2006.00159.x

Schade, J. (2005). Akzeptanz von Straßenbenutzungsgebühren: Entwicklung und Überprüfung eines Modells. Lengerich, Alemania: Pabst Science Publishers.

Schade, J. \& Schlag, B. (2003). Acceptability of transport pricing strategies. An introduction. En J. Schade \& \& B. Schlag (eds.), Acceptability of Transport Pricing Strategies (pp. 1-9). Oxford: Elsevier. https://doi.org/10.1108/9781786359506-001 
Schlag, B. \& Teubel, U. (1997). Public acceptability of traffic demand management in Europe. Traffic Engineering \& Control. 41(8), 314-318.

Thevathasan, A. \& Balachandran, B. (2007). Customers' perceptions of metropolitan train services in Melbourne. 30 th Australasian Transport Research Forum. Melbourne. En http://atrf.info/papers/2007/2007_Thevathasan_Balachandran.pdf

Thomson, I. (1982). Transporte urbano en América Latina. Consideraciones acerca de su igualdad y eficiencia. Revista de la CEPAL, 17 [E/CEPAL/G.1205], 85-117. En http:// repositorio.cepal.org/handle/11362/10387

Thomson, I. (1997). ¿ Por qué las inversiones en el transporte público no reducen la congestión de tránsito urbano? Revista de la CEPAL, 61 [LC/G.1955-P], 107-118. En http:// repositorio.cepal.org/bitstream/handle/11362/12049/1/061107118_es.pdf

Thomson, I. \& Bull, A. (2002). La congestión del tránsito urbano: causas y consecuencias económicas y sociales. Revista de la CEPAL, 76 [LC/L.1560-P], 109-121. En http:// www.cepal.org/es/node/30737

Transport for London (TfL). (2004). Congestion charging - Update on scheme impacts and operations. February 2004. Congestion charge publications and reports. Londres: Mayor of London / TfL. En http://www.cleanairinstitute.org/cops/bd/file/gdt/90-cc12monthson.pdf

Transport for London (TfL). (2008). Central London Congestion Charging - Impacts monitoring. Sixth Annual Report, July 2008. Congestion charge publications and reports. Londres: Mayor of London / TfL. En http://content.tfl.gov.uk/central-london-congestioncharging-impacts-monitoring-sixth-annual-report.pdf

Transport for London (TfL). (2015). Congestion Charge payments - Pay the Congestion Charge. TfL website. En https://tflgov.uk/modes/driving/congestion-charge/payingthe-congestion-charge

Tversky, A. (1972). Elimination by aspects: A theory of choice. Psychological Review, 79(4), 281-299. http://psycnet.apa.org/doi/10.1037/h0032955

Tyrinopoulos, Y. \& Antoniou, C. (2008). Public transit user satisfaction: Variability and policy implications. Transport Policy, 15(4), 260-272. https://doi.org/10.1016/j. tranpol.2008.06.002

Ubbels, B. J. \& Verhoef, E. T. (2004). Barriers to transport pricing. En P. Rietveld \& R. Stough (eds.), Barriers to sustainable transport: Institutions, regulation and sustainability (pp. 69-93). Londres / Nueva York: Spon Press/ Taylor \& Francis Group.

Vickrey, W. (1963). Pricing in urban and suburban transport. American Economic Review: 53(2) [Papers and Proceedings of the Seventy-Fifth Annual Meeting of the American Economic Association, May 1963], 452-465.

Walters, A. A. (1961). The theory and measurement of private and social cost of highway congestion. Econometrica, 29(4), 676-699. https://doi.org/10.2307/1911814

Wang, L., Xu, J. \& Qin, P. (2014). Will a driving restriction policy reduce car trips? The case study of Beijing, China. Transportation Research Part A: Policy and Practice, 67, 279290. https://doi.org/10.1016/j.tra.2014.07.014

Wolff, H. (2014). Keep your clunker in the suburb: Low-emission zones and adoption of green vehicles. The Economic Journal, 124(578), F481-F512. https://doi.org/10.1111/ ecoj.12091 